\title{
The differential expression of micro-RNAs 21, 200c, 204, 205, and 211 in benign, dysplastic and malignant melanocytic lesions and critical evaluation of their role as diagnostic biomarkers
}

\author{
Katherine Quiohilag ${ }^{1} \cdot$ Peter Caie $^{2} \cdot$ Anca Oniscu $^{1} \cdot$ Thomas Brenn $^{3} \cdot$ David Harrison $^{2}$ \\ Received: 23 October 2019 / Revised: 17 March 2020 / Accepted: 16 April 2020 / Published online: 9 May 2020 \\ (C) The Author(s) 2020
}

\begin{abstract}
Overlapping histological features between benign and malignant lesions and a lack of firm diagnostic criteria for malignancy result in high rates of inter-observer variation in the diagnosis of melanocytic lesions. We aimed to investigate the differential expression of five miRNAs $(21,200 \mathrm{c}, 204,205$, and 211) in benign naevi $(n=42)$, dysplastic naevi $(n=41)$, melanoma in situ $(n=42)$, and melanoma $(n=42)$ and evaluate their potential as diagnostic biomarkers of melanocytic lesions. Real-time PCR showed differential miRNA expression profiles between benign naevi; dysplastic naevi and melanoma in situ; and invasive melanoma. We applied a random forest machine learning algorithm to classify cases based on their miRNA expression profiles, which resulted in a ROC curve analysis of 0.99 for malignant melanoma and greater than 0.9 for all other groups. This indicates an overall very high accuracy of our panel of miRNAs as a diagnostic biomarker of benign, dysplastic, and malignant melanocytic lesions. However, the impact of variable lesion percentage and spatial expression patterns of miRNAs on these real-time PCR results was also considered. In situ hybridisation confirmed the expression of miRNA 21 and 211 in melanocytes, while demonstrating expression of miRNA 205 only in keratinocytes, thus calling into question its value as a biomarker of melanocytic lesions. In conclusion, we have validated some miRNAs, including miRNA 21 and 211, as potential diagnostic biomarkers of benign, dysplastic, and malignant melanocytic lesions. However, we also highlight the crucial importance of considering tissue morphology and spatial expression patterns when using molecular techniques for the discovery and validation of new biomarkers.
\end{abstract}

Keywords microRNA $\cdot$ Molecular pathology $\cdot$ Melanoma $\cdot$ Dermatopathology

\section{Introduction}

Malignant melanoma has seen a rapid increase in incidence in the last several decades, having risen by almost $50 \%$ in the last

Electronic supplementary material The online version of this article (https://doi.org/10.1007/s00428-020-02817-5) contains supplementary material, which is available to authorized users.

Katherine Quiohilag

k.quiohilag@nhs.net

1 Department of Pathology, Royal Infirmary of Edinburgh, 51 Little France Crescent, Edinburgh EH16 4SA, UK

2 School of Medicine, University of St Andrews, St Andrews, UK

3 Departments of Pathology \& Laboratory Medicine and Medicine and The Arnie Charbonneau Cancer Institute, Cumming School of Medicine, University of Calgary, Calgary, Canada decade alone, and is now the fifth most common cancer in the UK [1]. The current gold standard method for the diagnosis of melanocytic lesions is histological examination of tissue by pathologists. However, this has been described as 'one of the most challenging and controversial fields in diagnostic histopathology' [2] due to overlapping histological features between benign and malignant lesions [3] and lack of firm diagnostic criteria for malignancy. This creates the potential for both the over- and underdiagnosis of malignancy.

Dysplastic naevi represent a particular challenge. Histologically, they have overlapping features with both benign naevi at the mild end of the spectrum and melanoma at the severe end. Accordingly, various studies have shown high rates of interobserver variation in their histological diagnosis $[4,5]$. Of particular interest is a recent major study of 187 pathologists which concluded, in agreement with previous smaller studies, that diagnosis of dysplastic naevi and earlystage invasive melanoma was neither reproducible nor 
accurate [6]. Therefore, a reliable and objective molecular marker to aid pathologists' visual assessment of benign naevi, dysplastic naevi, and melanoma would be of great clinical value.

MicroRNAs (miRNAs) have been described as possessing 'most of the characteristics of an ideal biomarker': they are sensitive and specific [7] and are stable in FFPE (formalinfixed paraffin-embedded) tissue [8-11]. Over the last decade, the role of miRNAs as potential biomarkers in melanoma has attracted significant attention, with miRNAs found to have potential as diagnostic, prognostic, and predictive biomarkers, as well as putative therapeutic targets $[9,10,12-15]$.

miRNAs are short (up to 22 nucleotides), non-coding RNA molecules present in all cells. They function in posttranscriptional regulation of gene expression by binding to the 3' untranslated region (UTR) of mRNAs, blocking translation and sometimes accelerating mRNA degradation. Depending on their target, miRNAs can function as both tumour suppressors and tumour promoters. They provide a complex and powerful means of regulating gene expression, with computational predictions suggesting that they regulate onethird of the genome [16].

Although differential expression of various miRNAs has been demonstrated in benign and malignant melanocytic lesions, there is limited description of miRNA expression in dysplastic naevi and one of their major differential diagnoses, melanoma in situ [17-20], and studies which have been published tend to suffer from small cohort sizes. Given the significant degree of diagnostic uncertainly generated by these lesions, we aimed to investigate the role of a panel of five miRNAs as a potential diagnostic biomarker of benign naevi, dysplastic naevi, melanoma in situ, and invasive melanoma.

\section{Methods}

\section{Ethical approval and cohort selection}

Ethical approval for access to FFPE (formalin-fixed paraffinembedded) archival tissue was gained from the NRS BioResource and Tissue Governance Unit (SR961), and permission to conduct the study was gained from ACCORD via the Integrated Research Application System (IRAS). All cases were identified by searching the NHS Lothian Pathology archive. All diagnoses were confirmed by dermatopathologist review. Cases with insufficient lesional tissue for macrodissection and molecular analysis, defined in this study as lesions with a tumour percentage below $10 \%$, were excluded. The final cohort consisted of 42 cases of melanoma; 42 cases of melanoma in situ; 41 cases of dysplastic naevi; 42 cases of benign common naevi; and 17 cases of normal skin.

All morphological subtypes of melanoma with a vertical growth phase were included and detailed clinicopathological features were recorded (summarised in Table 1, with full details in ESM 1). Similarly, different morphological subtypes of melanoma in situ were included, with the final cohort consisting of 28 cases of melanoma in situ and 14 cases of lentigo maligna. Because of the known high level of diagnostic discordance of dysplastic naevi and the resulting potential for inconsistent use of this term between pathologists, inclusion criteria for this group were strict during dermatopathology review and were based on the WHO classification of skin tumours. All included cases had evidence of cytological atypia and displayed classical architectural features of dysplastic naevi including subepidermal fibroplasia; bridging of adjacent rete ridges; lentiginous proliferation of melanocytes; and shouldering in compound lesions. The final cohort consisted of 13 cases with mild atypia, 13 with moderate atypia, and 15 with severe atypia. Benign common naevi were intradermal or compound naevi without features of congenital type neavus.

All eligible cases were reviewed by a molecular pathologist and lesional areas for macrodissection were identified.

\section{Nucleic acid extraction}

Following microdissection, RNA was extracted using the Recoverall Total Nucleic Acid Isolation Kit for FFPE (AM1975, Thermo Fisher Scientific, UK) according to the manufacturer's protocol, with the exclusion of deparaffinisation steps. In summary, samples were digested in protease for $15 \mathrm{~min}$ at $50{ }^{\circ} \mathrm{C}$ (increased to $45 \mathrm{~min}$ for small lesions) followed by $15 \mathrm{~min}$ at $80^{\circ} \mathrm{C}$. Samples were incubated with DNase mix for $30 \mathrm{~min}$ at room temperature prior to further washing and elution. The concentration of RNA extract was measured using a Qubit Fluorometer with the high sensitivity RNA assay.

\section{Reverse transcription and real-time PCR}

miRNA expression levels were assessed using the TaqMan ${ }^{\circledR}$ small RNA assay protocol from Applied Biosystems. Assays specific to miR-21 (hsa-miR-21, assay ID 00397); miR-200c (hsa-miR-200c, assay ID 002300); miR-204 (hsa-miR-204, assay ID 000508); miR-205 (hsa-miR-205, assay ID 000509); and miR-211 (hsa-miR-211, assay ID 000514) were used, with U6 (RNU6B, assay ID 001093) as an endogenous control (Thermo Fisher Scientific). Validation experiments were performed for each assay with serial tenfold dilutions of one sample, run in triplicate, to ensure the amplification efficiency of the endogenous control (U6) and each target miRNA was approximately equal. For all miRNA assays, the plot of $\triangle \mathrm{CT}$ against log input resulted in a semi-log regression line with a slope $<0.1$.

Reverse transcription was carried out using the TaqMan ${ }^{\mathrm{TM}}$ MicroRNA Reverse Transcription Kit (catalogue number 
Table 1 Summary of clinicopathological features of melanoma cohort. LVI, lymphovascular invasion

\begin{tabular}{|c|c|c|c|c|c|c|c|c|}
\hline $\begin{array}{l}\text { Morphological } \\
\text { subtype }\end{array}$ & $\begin{array}{l}\text { Median } \\
\text { Breslow } \\
\text { thickness }\end{array}$ & $\begin{array}{l}\text { Mean mitotic } \\
\text { count }\left(\text { per } \mathrm{mm}^{2} \text { ) }\right.\end{array}$ & $\begin{array}{l}\text { Number with } \\
\text { ulceration }\end{array}$ & $\begin{array}{l}\text { Number with } \\
\text { regression }\end{array}$ & $\begin{array}{l}\text { Number with } \\
\text { precursor naevus }\end{array}$ & $\begin{array}{l}\text { Number } \\
\text { with LVI }\end{array}$ & $\begin{array}{l}\text { Number with } \\
\text { micro-satellites }\end{array}$ & $\begin{array}{l}\text { Immune } \\
\text { response }\end{array}$ \\
\hline 18 nodular & 3.6 & 7 & 18 & 5 full & 3 & 3 & 0 & 2 brisk \\
\hline $\begin{array}{l}18 \text { superficial } \\
\text { spreading }\end{array}$ & & & & 1 partial & & & & $\begin{array}{l}15 \\
\text { non-- } \\
\text { brisk }\end{array}$ \\
\hline $\begin{array}{l}2 \text { desmoplastic } 2 \\
\text { acral lentiginous } \\
1 \text { lentigo maligna } \\
\text { melanoma }\end{array}$ & & & & & & & & 25 absent \\
\hline $\begin{array}{l}1 \text { desmoplastic and } \\
\text { spindle cell }\end{array}$ & & & & & & & & \\
\hline
\end{tabular}

4366596, Thermo Fisher Scientific). All RNA samples were diluted in nuclease-free water to $1 \mathrm{ng}$ in $5 \mu \mathrm{l}$, with each $15 \mu \mathrm{l}$ of reverse transcription reaction consisting of $5 \mu \mathrm{l}$ of RNA, $7 \mu \mathrm{l}$ of reverse transcription mastermix, and $3 \mu \mathrm{l}$ of reverse transcription primer.

Real-time PCR was carried out on 96-well plates using TaqMan ${ }^{\circledR}$ Universal PCR Master Mix II, No UNG (catalogue number 4440043, Thermo Fisher Scientific) with each 20- $\mu 1$ PCR reaction made up as follows: $1-\mu$ l TaqMan small RNA assay; $1.33-\mu l$ product from reverse transcription reaction; $10-\mu 1$ PCR mastermix; and 7.67- $\mu 1$ nuclease-free water. Samples were run on an Applied Biosystems ${ }^{\mathrm{TM}} 7500$ SDS real-time PCR machine (Thermo Fisher Scientific). All reactions were carried out in duplicate with negative controls.

\section{In situ hybridisation}

Chromogenic in situ hybridisation was carried out on a subset of cases (benign naevi $(n=6)$, dysplastic naevi $(n=6 ; 3$ with mild atypia, 1 with moderate atypia, 2 with severe atypia), melanoma in situ $(n=6)$, and melanoma $(n=6))$ using BaseScope $^{\mathrm{TM}}$ assays (ACDbio) specific to each miRNA of interest according to the manufacturer's protocol. PPIB probes were used as positive controls and DAPB as negative controls (ESM 2 and 3).

In summary, for pre-treatment, slides were incubated with 5-8 drops of hydrogen peroxide at room temperature for $10 \mathrm{~min}$ and bathed in antigen retrieval solution within the Braun Multiquick FS 20 steamer for 15 min. Following air drying, a hydropic barrier was drawn around the tissue edge. The next day, the slides were incubated with protease IV at $40{ }^{\circ} \mathrm{C}$ for $45 \mathrm{~min}$, with re-application of the protease after $30 \mathrm{~min}$, followed by incubation with the relevant probe at $40{ }^{\circ} \mathrm{C}$ for $2 \mathrm{~h}$. The probes were amplified by the addition of AMPS 0-6 in turn as follows: AMP 0 for $30 \mathrm{~min}$ at $40{ }^{\circ} \mathrm{C}$; AMP 1 for 15 min at $40^{\circ} \mathrm{C}$; AMP 2 for 30 min at $40^{\circ} \mathrm{C}$; AMP 3 for 30 min at $40{ }^{\circ} \mathrm{C}$; AMP 4 for 15 min at $40{ }^{\circ} \mathrm{C}$; AMP 5 for
45 min at room temperature; and AMP 6 for 15 min at room temperature. Slides were then incubated with RNAScope fast RED solution for $10 \mathrm{~min}$ at room temperature and counterstained with haematoxylin and lithium carbonate. Following drying overnight, the spatial distribution of transcripts was assessed by two independent pathologists.

\section{Statistical analysis}

The comparative CT method was applied to calculate $\Delta \Delta \mathrm{CT}$ and expression fold changes in each miRNA. If the fold change was between 0 and 1 , indicating negative fold change in expression, the formula $-1 / 2-\Delta \Delta \mathrm{CT}$ was used for linear transformation. Statistical analysis of real-time PCR results was performed using MiniTab version 17. Relative expression of each miRNA in each sample was calculated using the formula 2- $\Delta \mathrm{CT}$, followed by $\log$ transformation (base 2). Normality was confirmed with the Anderson-Darling tests and probability plots. Welch's ANOVA followed by the Games-Howell pairwise comparison (with 95\% confidence intervals) was used to compare the expression levels of each miRNA between groups. Adjusted $p$ values are provided for pairwise comparisons. Tests of equal variance followed by 1way ANOVA and Tukey's pairwise comparison were used to compare the expression levels of each miRNA in dysplastic naevi with mild, moderate, and severe atypia. A two-tailed $t$ test was used to compare expression patterns in different morphological subtypes of melanoma and melanoma in situ. Correction for lesion percentage was performed by dividing relative expression by the lesion percentage prior to $\log$ transformation.

\section{Decision tree modelling}

Random forest with 100 trees was performed with logtransformed relative expression data using Weka opensource machine learning software version 3.6.13. Tenfold 
cross-validation was used to avoid overfitting. Receiver operating characteristic (ROC) curve analysis was used to evaluate overall accuracy as a diagnostic test.

\section{Results}

\section{Expression of miRNAs in benign naevi, dysplastic naevi, melanoma in situ, and melanoma}

The expression of miRNA 21 was significantly increased in melanoma compared with that in all other groups $(p<0.001)$. Conversely, there was a significant reduction in the expression of both miRNA 200c and 205 in melanoma compared with that in all other groups $(p<0.001)$. The expression of miRNA 204 and 211 was significantly higher in benign naevi compared with that in all other groups $(p<0.001)$ (Fig. 1). The expression of miRNA 200c also showed significantly lower expression in melanoma in situ compared with that in dysplastic naevi $(p<0.05)$ and benign naevi $(p<0.05)$. For miRNA 211, expression was significantly reduced in melanoma in situ compared with that in dysplastic naevi $(p<0.001)$, though owing to a wide variation in expression in the melanoma cohort, neither of these was significantly different from melanoma. There were no significant differences in the expression of miRNA 21 and 205 in benign naevi, dysplastic naevi, and melanoma in situ, and no significant differences in the expression of miRNA 204 in dysplastic naevi, melanoma in situ, and melanoma (Fig. 1).

Examination of miRNA expression in the form of fold changes reveals a 3.41-fold increase in miRNA 21 in melanoma compared with that in benign naevi, with almost 8and 7-fold reduced expression of miRNA 200c and 205, respectively (Table 2). The greatest fold changes are seen in miRNA 204 and 211, with almost 15- and 16-fold reduced expression respectively in melanoma compared with that in benign naevi. Large negative fold changes in the expression of miRNAs-204 and 211 are also seen in dysplastic naevi and melanoma in situ compared with those in benign naevi.

Using 1-way ANOVA with Tukey's pairwise comparisons, there was no significant difference between the expression levels of all five miRNAs in dysplastic naevi with mild, moderate and severe atypia. Two-tailed $t$ tests showed no significant differences in the expression of any miRNAs between different morphological subtypes of malignant melanoma or melanoma in situ.

Overall, dysplastic naevi and melanoma in situ showed a similar pattern of expression, generally grouping with benign naevi for miRNAs $21,200 \mathrm{c}$, and 205, and with melanoma for miRNAs 204 and 211.

\section{Expression of miRNAs in normal skin}

miRNAs 21, 200c, and 205 showed a similar pattern of expression to that seen in benign and dysplastic naevi and were significantly different from melanoma (Fig. $1 ; p<0.001$ ). miRNA 200c expression in normal skin was also significantly higher than that of melanoma in situ $(p<0.05)$. miRNA 204 and 211, however, showed significantly lower levels of expression in normal skin than they did in benign naevi and dysplastic naevi $(p<0.001)$. miRNA 211 expression in normal skin was also significantly lower than that in the melanoma in situ and melanoma cohorts $(p<0.001)$, as was miRNA 204 expression but to a slightly lesser extent $(p<0.05)$.

\section{Decision tree modelling}

Random forest was able to correctly classify 36 out of 42 melanomas following tenfold cross-validation (Table 3 ). Four of the six false-negative melanomas were classified as melanoma in situ, resulting in only two cases which were incorrectly classified as benign. There was one false-positive melanoma which was a melanoma in situ, meaning no benign lesions were incorrectly classified as malignant. Random forest gave very high ROC areas of 0.99 for melanoma, 0.97 for benign naevi, 0.90 for dysplastic naevi, and 0.91 for melanoma in situ, indicating overall high accuracy of this panel of miRNAs as a diagnostic test.

\section{The impact of lesion percentage}

One limitation of molecular testing with real-time PCR is that, despite macrodissection, cell types other than melanocytes will inevitably be incorporated into the samples. This effect will be greater in cases with a low lesion percentage. After macrodissection, the average lesion percentage for the dysplastic naevus cohort was $30 \%$ and $20.5 \%$ for the melanoma in situ cohort. This compared to $65.6 \%$ for the melanoma cohort and $62.5 \%$ for the benign naevus cohort. Despite the likely impact of lesion percentage on the measured levels of the miRNAs with real-time PCR, no adjustment for this was identified in the published literature.

Following correction for lesion percentage, expression of miRNA 21 remains significantly lower in benign naevi compared to all other groups $(p<0.05)$, with expression in dysplastic naevi also significantly lower than melanoma and melanoma in situ ( $p<0.05$, Fig. 2). Expression of both miRNA $200 \mathrm{c}$ and 205 remains significantly lower in melanoma compared with that in all three other groups $(p<0.001)$, though following correction expression is higher in dysplastic naevi and melanoma in situ compared with that in benign naevi $(p<0.005)$. Corrected expression of miRNA 204 and 211 was significantly lower in melanoma compared with that in all other groups $(p<0.005)$ and significantly higher in benign 
a
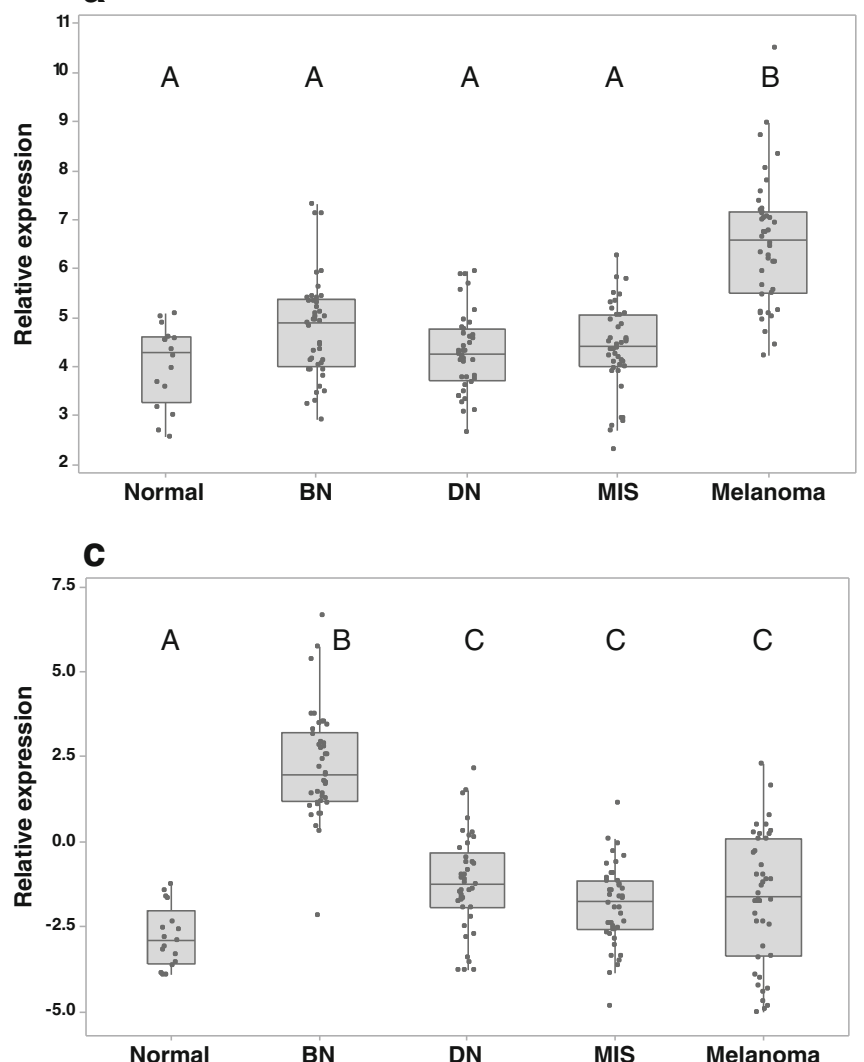

e

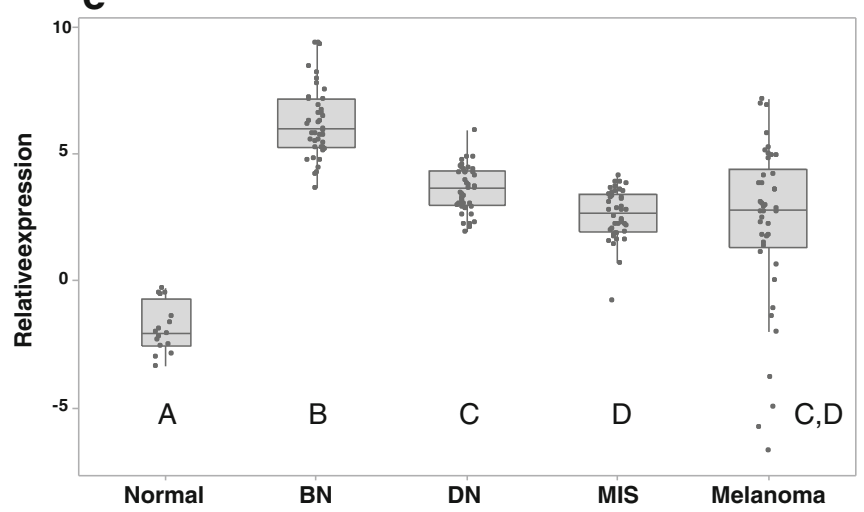

Fig. 1 Boxplots of log-transformed relative expression of microRNAs measured with real-time PCR. miRNAs 21 (a); 200c (b); 204 (c); 205 (d); and 211 (e) in normal skin, benign naevi (BN), dysplastic naevi (DN), melanoma in situ (MIS), and melanoma. Letters indicate outcome of

naevi compared with that in all other groups $(p<0.001)$, with intermediate expression in dysplastic naevi and melanoma in

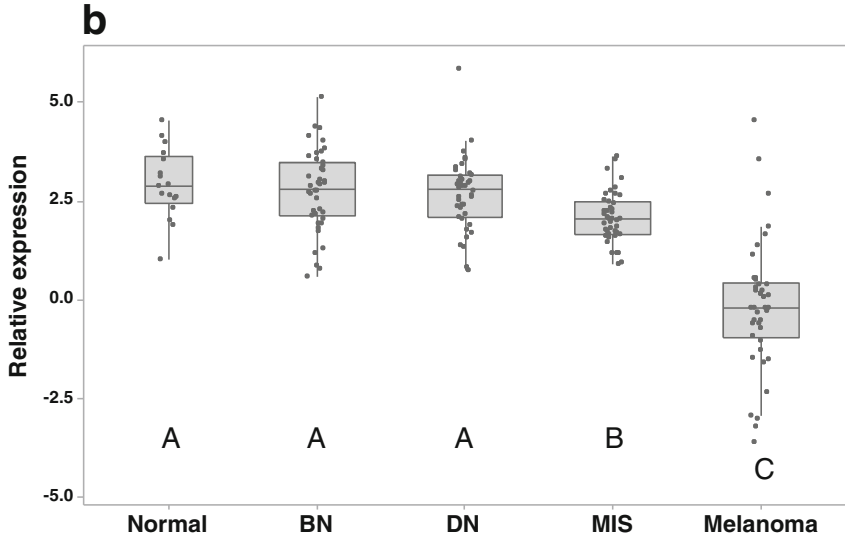

d

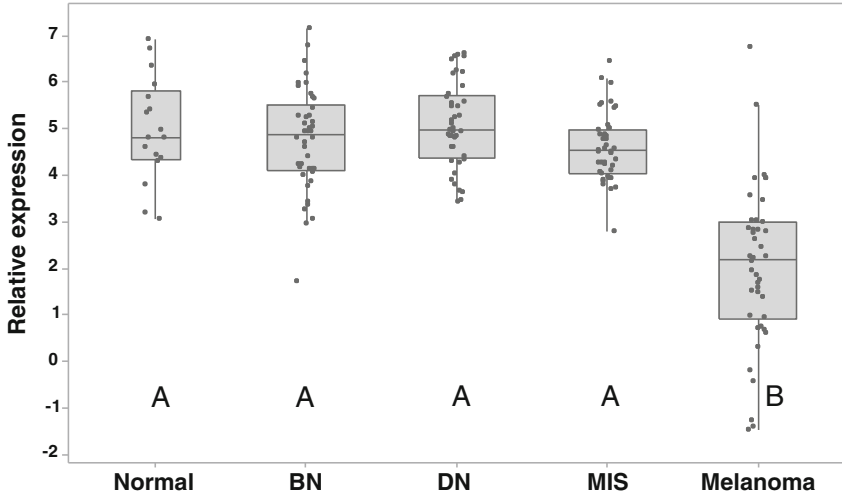

Welch's ANOVA followed by Games-Howell pairwise comparisons. Groups which do not share a letter are significantly different from each other $(p<0.05)$

situ. Random forest was able to correctly classify 37 out of 42 melanomas and 39 out of 42 benign naevi (Table 4). There

Table 2 Fold changes in expression of each micro-RNA in dysplastic naevi, melanoma in situ, and melanoma compared with benign naevi

\begin{tabular}{lccccc}
\hline & mRNA 21 & miRNA 200c & miRNA 204 & miRNA 205 & miRNA 211 \\
\hline Dysplastic naevi & -1.39 & -1.07 & -11.04 & 1.20 & -6.33 \\
Melanoma in situ & -1.30 & -1.58 & -16.99 & -1.12 & -12.56 \\
Melanoma & 3.41 & -7.57 & -14.96 & -6.66 & -15.96 \\
\hline
\end{tabular}


Table 3 Confusion matrix from random forest with tenfold crossvalidation. Each horizontal row shows how cases from each group ( $B N$, benign naevi; $D N$, dysplastic naevi; $M I S$, melanoma in situ) were classified based on the miRNA expression data

\begin{tabular}{llllll}
\hline & $\begin{array}{l}\text { Classified as } \\
\text { normal }\end{array}$ & $\begin{array}{l}\text { Classified as } \\
\text { BN }\end{array}$ & $\begin{array}{l}\text { Classified as } \\
\text { DN }\end{array}$ & $\begin{array}{l}\text { Classified as } \\
\text { MIS }\end{array}$ & $\begin{array}{l}\text { Classified as } \\
\text { melanoma }\end{array}$ \\
\hline Normal & 16 & 0 & 1 & 0 & 0 \\
BN & 0 & 39 & 2 & 1 & 0 \\
DN & 0 & 4 & 27 & 10 & 0 \\
MIS & 1 & 1 & 11 & 28 & 1 \\
Melanoma & 0 & 2 & 0 & 4 & 36 \\
\hline
\end{tabular}
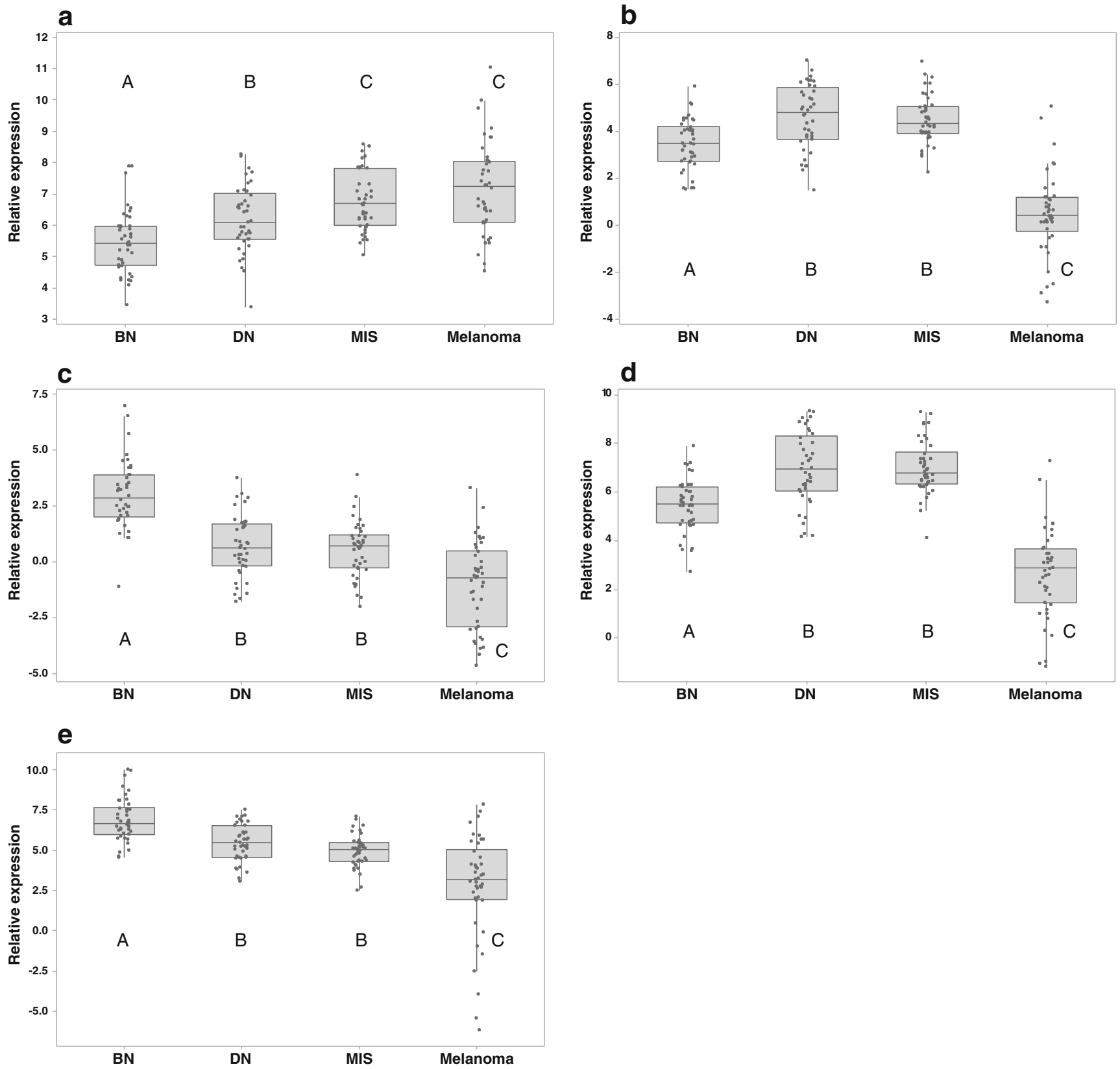

Fig. 2 Boxplots of log-transformed relative expression of microRNAs measured with real-time PCR following correction for lesion percentage. miRNAs 21 (a); 200c (b); 204 (c); 205 (d); and 211 (e) in benign naevi

Letters indicate outcome of Welch's ANOVA followed by Games-Howell pairwise comparisons. Groups which do not share a letter are significantly different from each other $(p<0.05)$ 
Table 4 Confusion matrix from random forest with tenfold crossvalidation following correction for lesion percentage. Each horizontal row shows how cases from each group $(B N$, benign naevi; $D N$, dysplastic naevi, $M I S$, melanoma in situ) were classified based on the miRNA expression data

\begin{tabular}{lcccc}
\hline & Classified as BN & Classified as DN & Classified as MIS & Classified as melanoma \\
\hline BN & 39 & 3 & 0 & 0 \\
DN & 3 & 27 & 10 & 1 \\
MIS & 1 & 13 & 27 & 1 \\
Melanoma & 1 & 1 & 3 & 37 \\
\hline
\end{tabular}

were only two false-positive melanomas, one of which was a melanoma in situ, and only two malignant lesions were misclassified as benign naevi, one melanoma in situ, and one melanoma. The overall ROC areas remained very high for benign naevi and melanoma, at 0.98 and 0.97 , respectively, and high for dysplastic naevi and melanoma in situ at 0.86 and 0.89 , respectively.

\section{In situ hybridisation}

An important consideration with the above approach to correcting for lesion percentage is that it assumes expression of all miRNAs in lesional cells only, with differences between groups reflective of differential expression in lesional cells. To further assess the spatial expression of each miRNA, in situ hybridisation with BaseScope ${ }^{\mathrm{TM}}$ was performed on a subset of cases. Due to low sensitivity of the probes resulting from the short target sequences (direct communication with ACDbio) and variable fixation times of clinical samples, we do not believe the results of in situ hybridisation to be accurately and reliably quantifiable. This technique has therefore only been applied to demonstrate spatial expression of miRNAs, and not as a method of quantifying miRNA expression.

Despite assay optimisation, the expression of miRNA 200c and 204 remained too low to be reliably and consistently visualised. miRNA 21 was widely expressed in invasive malignant melanocytes, with minimal expression in background keratinocytes (Fig. 3a). miRNA 205 was consistently expressed in keratinocytes in both benign and malignant lesions, with minimal expression detected in benign and malignant melanocytes (Fig. 3b). miRNA 211, on the other hand, was consistently expressed in benign and malignant melanocytes, with minimal expression detected in keratinocytes (Fig. 3c). No miRNA expression was identified in other background cell types including lymphocytes and stromal cells.

\section{Discussion}

Our study has demonstrated significantly increased expression of miRNA 21 and reduced expression of miRNAs 200c, 204, 205, and 211 in melanoma compared with that in benign naevi. This is consistent with previously published data $[9$,
12, 14, 15]. The expression profiles of miRNA 21 and 211 in dysplastic naevi, using a significantly larger sample size than previously published studies, are also largely consistent with previously published results [18-20]. One exception is a previous study which used fluorescent in situ hybridisation to show high miRNA 211 expression was maintained in a cohort of 21 dysplastic naevi, regardless of the degree of atypia, with significantly reduced expression in invasive melanoma [17]. We also demonstrated high sensitivity and specificity of this miRNA panel as a potential diagnostic test using a random forest machine learning algorithm.

These results, however, should be interpreted in the context of possible study limitations. It is recognised that both melanoma and melanoma in situ encompass a group of morphologically and biologically diverse lesions. We included a cohort representative of the diversity of melanoma typically encountered in diagnostic pathology practice, within which we did not find any significant differences in miRNA expression between different morphological subtypes. However, this may be appropriate for further analysis with a larger melanoma cohort including selection for rarer subtypes.

In addition, it is noted that, despite the high ROC areas resulting from the random forest algorithm, there remains a level of misclassification between dysplastic naevi and melanoma in situ which may impact clinical utility as a diagnostic test. When the miRNA expression data was scrutinised in greater detail, it was considered likely that variable lesion percentages in different groups had an impact on the final expression profiles. Correction for lesion percentage resulted in miRNAs 21, 204, and 211 showing an intermediate level of expression in dysplastic naevi and melanoma in situ, in between that of benign naevi and melanoma. Furthermore, miRNA 21 showed significantly increased levels of expression from benign naevi to dysplastic naevi to melanoma in situ, with melanoma in situ showing no significant difference from melanoma. This provides further evidence of a correlation with increasing degrees of atypia, as has been proposed previously [20]. For miRNAs 200c and 205, however, correction for lesion percentage resulted in dysplastic naevi and melanoma in situ showing a higher expression pattern than both melanoma and benign naevi, a finding which does not have any obvious biological explanation. 
Fig. 3 miRNA 21 (a), miRNA 205 (b), and miRNA 211 (c) expression in melanoma and benign naevus samples using BaseScope. a High expression of miRNA 21 in invasive malignant melanocytes with minimal expression in benign melanocytes, both with minimal expression in background keratinocytes. b Expression of miRNA 205 in keratinocytes, with minimal expression in melanocytes. $\mathbf{c}$ Expression of miRNA 211 in melanocytes, with no significant expression in keratinocytes. Black lines represent the approximate division between keratinocytes and melanocytes. All photos $\times 20$ magnification

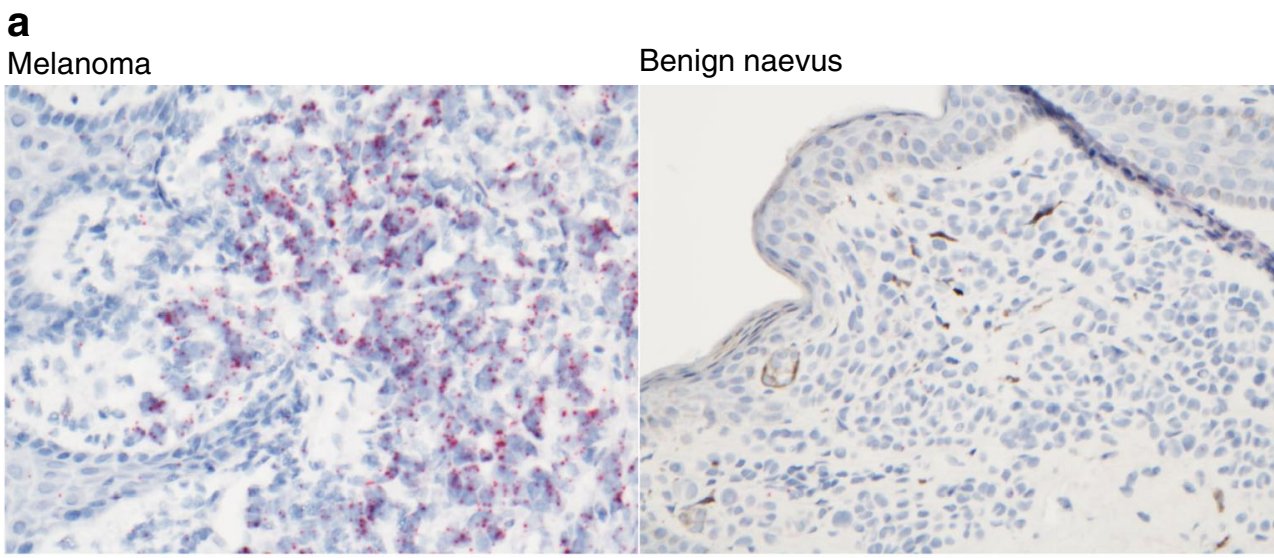

b

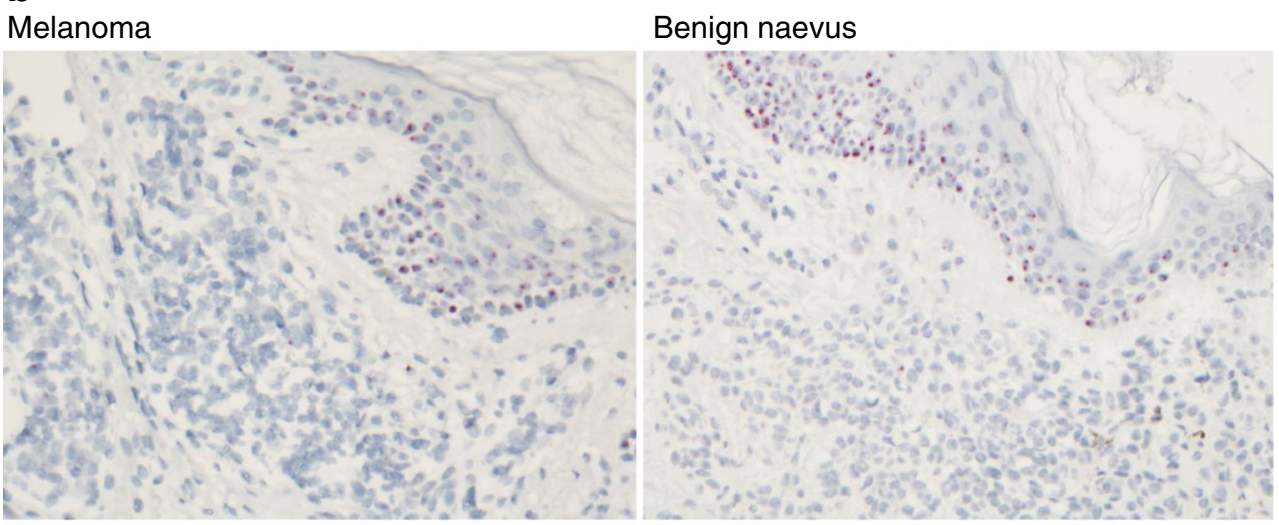

C

Melanoma

Benign naevus

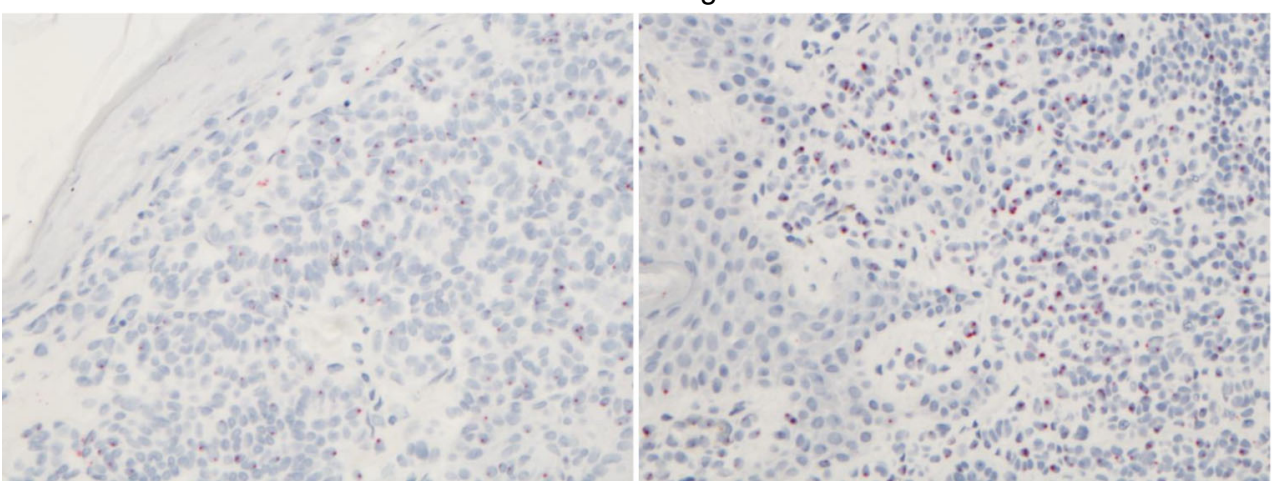

A further explanation for the corrected expression profiles can be sought by examining expression patterns in normal skin. Low expression of miRNA 204 and 211 in normal skin compared with that of benign naevi suggests expression only in melanocytes (which are found in low numbers in normal skin). Conversely, a similar expression of miRNA 200c and 205 in normal skin and benign naevi suggests expression in additional cell types such as keratinocytes. If there is, as suspected, expression of some of the miRNAs in background cells such as keratinocytes, this would clearly have an impact on expression levels of miRNAs measured by real-time PCR.
It could also result in over-correction for lesion percentage, as some or all of the measured miRNA would be originating from background cells.

This hypothesis was tested using in situ hybridisation, which demonstrated expression of miRNA 21 and 211 principally in melanocytes, with minimal expression detected in keratinocytes. miRNA 205, however, was primarily expressed in keratinocytes, with minimal expression detected in melanocytes. Given the identical expression profiles of miRNA 200c and 205 with PCR, it is speculated that miRNA 200c may also be more highly 
expressed in keratinocytes than melanocytes. Similarly, given the identical expression profiles of miRNA 211 and 204, it is speculated that miRNA 204 may be expressed principally in melanocytes.

Therefore, although miRNA 205 expression is clearly associated with melanoma, the reduced expression in melanoma compared with that in all other lesion types with real-time PCR could be explained by the reduced proportion of keratinocytes in melanoma samples. Similarly, the expression in keratinocytes would account for the relatively high corrected expression in dysplastic naevi and melanoma in situ. With miRNA 21 and miRNA 211 expressed principally in melanocytes, their function as a potential biomarker of melanocytic lesions is verified. However, this also makes it difficult to be certain, when lesion percentage is not accounted for, whether low expression in dysplastic naevi and melanoma in situ with real-time PCR is due to downregulation with atypia or malignant transformation, or due to low proportion of melanocytes in these samples, or a combination of the two.

One recently published paper of particular interest examined miRNA expression in keratinocyte, melanocyte, and melanoma cell lines [15]. miRNA 204 and 211 showed significantly higher expression in cultured melanocytes than in cultured keratinocytes, while miRNA 200c and 205 showed significantly higher expression in cultured keratinocytes than in cultured melanocytes. Our study provides further support of these results by showing miRNA 211 expression principally in melanocytes and miRNA 205 expression principally in keratinocytes in human tissue samples.

The absence of significant expression of miRNA 205 in melanocytes could have considerable implications for the interpretation of the literature suggesting a role as a biomarker of melanoma, particularly as most papers examining miRNA expression profiles use techniques which result in loss of spatial expression, including miRNA microarrays, real-time PCR, and deep sequencing. Therefore, while supporting the potential for specific miRNAs including miRNA 21 and 211 to act as biomarkers of melanocytic lesions, we also highlight the crucial importance of consideration of tissue morphology and spatial expression prior to application of molecular techniques in the discovery and validation of biomarkers using human tissue specimens.

Acknowledgements We thank Dr. Kathy Walsh for her technical assistance with real-time PCR and Dr. Jenna Gregory for her technical assistance with in situ hybridisation.

Contribution statement $\mathrm{KQ}, \mathrm{AO}, \mathrm{DH}, \mathrm{PC}$, and TB conceived and designed the study. KQ wrote, edited, and reviewed the manuscript, with TB and $\mathrm{AO}$ also editing and reviewing the manuscript. KQ, $\mathrm{AO}$, and TB examined the slides. KQ carried out qRT-PCR and in situ hybridisation. PC provided statistical support and carried out decision tree modelling. All authors gave final approval for publication. KQ takes responsibility for the work as a whole, including the study design, access to data, and the decision to submit and publish the manuscript.

\section{Compliance with ethical standards}

Ethical approval for access to FFPE (formalin-fixed paraffin-embedded) archival tissue was gained from the NRS BioResource and Tissue Governance Unit (SR961), and permission to conduct the study was gained from ACCORD via the Integrated Research Application System (IRAS).

Conflict of interest The authors declare that they have no conflict of interest.

Open Access This article is licensed under a Creative Commons Attribution 4.0 International License, which permits use, sharing, adaptation, distribution and reproduction in any medium or format, as long as you give appropriate credit to the original author(s) and the source, provide a link to the Creative Commons licence, and indicate if changes were made. The images or other third party material in this article are included in the article's Creative Commons licence, unless indicated otherwise in a credit line to the material. If material is not included in the article's Creative Commons licence and your intended use is not permitted by statutory regulation or exceeds the permitted use, you will need to obtain permission directly from the copyright holder. To view a copy of this licence, visit http://creativecommons.org/licenses/by/4.0/.

\section{References}

1. Skin Cancer Statistics 2017 [http://www.cancerresearchuk.org/ health-professional/cancer-statistics/statistics-by-cancer-type/skincancer]

2. Brenn T. Pitfalls in the evaluation of melanocytic lesions. Oxford, UK2012. p. 690-705

3. Urso C, Rongioletti F, Innocenzi D, Batolo D, Chimenti S, Fanti PL, Filotico R, Gianotti R, Lentini M, Tomasini C, Pippione M (2005) Histological features used in the diagnosis of melanoma are frequently found in benign melanocytic naevi. J Clin Pathol 58(4):409-412

4. Brochez L, Verhaeghe E, Grosshans E, Haneke E, Piérard G, Ruiter D, Naeyaert JM (2002) Inter-observer variation in the histopathological diagnosis of clinically suspicious pigmented skin lesions. J Pathol 196(4):459-466

5. Lyn MD, Marianne B, Jan AB, Byers HR, Martin CM, Raymond LB (1993) Histopathologic recognition and grading of dysplastic melanocytic nevi: an Interobserver agreement study. J Investig Dermatol 100(3s):318S

6. Elmore JG, Barnhill RL, Elder DE, Longton GM, Pepe MS, Reisch LM et al (2017) Pathologists' diagnosis of invasive melanoma and melanocytic proliferations: observer accuracy and reproducibility study. BMJ (Clinical research ed) 357:j2813

7. Detassis S, Grasso M, Del Vescovo V, Denti MA (2017) microRNAs make the call in cancer personalized medicine. Front Cell Dev Biol 5

8. Varamo C, Occelli M, Vivenza D, Merlano M, Lo Nigro C. MicroRNAs role as potential biomarkers and key regulators in melanoma. 2017. p. 3-10

9. Latchana N, Ganju A, Howard JH, Carson WE (2016) MicroRNA dysregulation in melanoma. Surg Oncol 25(3):184-189

10. Luo C, Weber CEM, Osen W, Bosserhoff A-K, Eichmüller SB (2014) The role of microRNAs in melanoma. Eur J Cell Biol 93(1-2):11-22 
11. Philippidou D, Schmitt M, Moser D, Margue C, Nazarov PV, Muller A, Vallar L, Nashan D, Behrmann I, Kreis S (2010) Signatures of microRNAs and selected microRNA target genes in human melanoma. Cancer Res 70(10):4163-4173

12. Mirzaei H, Gholamin S, Shahidsales S, Sahebkar A, Jafaari MR, Mirzaei HR et al (2016) MicroRNAs as potential diagnostic and prognostic biomarkers in melanoma. Eur J Cancer 53:25-32

13. Thyagarajan A, Shaban A, Sahu RP (2018) MicroRNA-directed cancer therapies: implications in melanoma intervention. $\mathrm{J}$ Pharmacol Exp Ther 364(1):1-12

14. Xu Y, Brenn T, Brown ERS, Doherty V, Melton DW (2012) Differential expression of microRNAs during melanoma progression: miR-200c, miR-205 and miR-211 are downregulated in melanoma and act as tumour suppressors. Br J Cancer 106(3):553-561

15. Galasso M, Morrison C, Minotti L, Corrà F, Zerbinati C, Agnoletto C, Baldassari F, Fassan M, Bartolazzi A, Vecchione A, Nuovo GJ, di Leva G, D'Atri S, Alvino E, Previati M, Nickoloff BJ, Croce CM, Volinia S (2018) Loss of miR-204 expression is a key event in melanoma. Mol Cancer 17(1):71
16. Sun V, Zhou WB, Majid S, Kashani-Sabet M, Dar AA (2014) MicroRNA-mediated regulation of melanoma. Br J Dermatol 171(2):234-241

17. Babapoor S, Horwich M, Wu R, Levinson S, Gandhi M, Makkar H et al (2016) microRNA in situ hybridization for miR-211 detection as an ancillary test in melanoma diagnosis. Mod Pathol 29(5):461

18. Babapoor S, Wu R, Kozubek J, Auidi D, Grant-Kels JM, Dadras SS (2017) Identification of microRNAs associated with invasive and aggressive phenotype in cutaneous melanoma by next-generation sequencing. Lab Investig 97(6):636

19. James K, Zhihai M, Elizabeth F, Tatiana D, Rong W, Dong-Guk S et al In-depth characterization of microRNA transcriptome in melanoma. PLoS ONE 8(9):e72699

20. Jiang L, Lv X, Li J, Li J, Li X, Li W, Li Y (2012) The status of microRNA-21 expression and its clinical significance in human cutaneous malignant melanoma. Acta Histochem 114(6):582-588

Publisher's note Springer Nature remains neutral with regard to jurisdictional claims in published maps and institutional affiliations. 